\title{
Face Recognition System Using Hybrid Approach of Both Principal Component Analysis and Linear Discriminant Analysis
}

\author{
Priyanka Sood $^{1}$, Er. Shivali Puri ${ }^{2}$, Er. Varinderjit Kaur ${ }^{3}$, Dr. Naveen Dhillon ${ }^{4}$ \\ Dept. of Computer Science and Engg, Ramgarhia Institute of Engg and Tech, Phagwara, Punjab ${ }^{1}$ \\ Assistant Prof., Dept. of Computer Science and Engg, Ramgarhia Institute of Engg and Tech, Phagwara, Punjab ${ }^{2}$ \\ H.O.D (M.Tech), Dept. of Computer Science and Engg, Ramgarhia Institute of Engg and Tech, Phagwara, Punjab \\ Principal, Ramgarhia Institute of Engg and Tech, Phagwara, Punjab ${ }^{4}$
}

\begin{abstract}
Face recognition is emerging as one of the popular field in the biometric research. It is used in various surveillance systems for the security purpose as it doesn't need object comparison. The main advantage of using face recognition system is its uniqueness and acceptance over other biometric systems. Though this system is considered to be accurate but the detection of the face is a difficult process due to the high degree of variability in faces. Face recognition is a section of pattern recognition in which human visual perception is saved in the computer. Many researchers are working on this field for many of the years, many algorithms and techniques are developed to update the traditional systems. Some of the techniques that are common these days are PCA, LDA, and Gabor etc. But these approaches individually are not that much efficient in some of the cases. In this research work a new approach is proposed, in which the main concentration is to extract the features with the PCA and LDA techniques as well as the combined systems. This is a much better approach to work with, as suggested from the literature and also more successful for the large dataset. After combining both of the feature extraction, finally the classification will be done and the performance will be evaluated.
\end{abstract}

Keywords: Face Recognition, Biometric System, Linear Discriminant Analysis, Principal Component Analysis.

\section{INTRODUCTION}

Biometric recognition links the person to his/her previously established identity through automated means, therefore, the result is based on those pre-defined parameters. Normally the operations of the biometric system are divided into two stages as Follows:

- Enrollment

- Recognition

Enrollment is a Stage in which the biometric information related to a person is stored in a database. This reference information can be in the form of templates or biometric sample itself. Templates contain the extracted features from the biometric sample or parameters of mathematical models that best characterizes the extracted features. In some application biometric parameters such as name, id is also stored along with biometric references. In the case of loss of identity, information such as unknown latent prints lifted from the crime scene, anonymous authentication etc. The reference is used with system generated Id for future use. In Recognition Stage, firstly the scanning of users biometric traits is done, Secondly extraction takes place and then the process of Matching is performed corresponding to the reference biometric information stored in the database. A high similarity score between the query and the reference data results in the user being authenticated or identified. Facial recognition is not reliable and efficient as compared to the other recognition techniques. Whereas there is an advantage that it has no need to have consent from the test subject. Properly designed system which is installed on airports and multiplexes are able to recognize even the single person in the crowd of thousands. Whereas the techniques like iris recognition, fingerprints recognition is not able to perform such kind of detection from such a huge crowd. However, the question is that whether the face recognition systems can generate a reliable or efficient result in the case of railways stations security and airport security.

\section{Techniques}

Some facial algorithms are used to analyze the extracted portion from the inputted image. e.g. an algorithm is used to the relative position, size, and/or shape of the eyes, nose, cheekbones, and jaw. The same features are then used for other images of same or matched patterns. Other algorithm is used to normalize the data of images in form of gallery and then archives this face data but saves only those images which are important or useful for recognition. Then the face data is compared with an analyzed image. The traditional system was based on template matching technique and by using this compressed 
image was created to represent a specific portion or feature of the face. List of some of the techniques is as follows:

- DWT (Discrete Wavelet Transform) a frequency domain feature extraction technique. In this method the one-dimensional signal is divided into two parts, one is the high frequency and other is the low-frequency part. The edge components of the signal are present in the higher frequency part. The low-frequency part is split into two parts and a similar process will be continued till the desired level is achieved. In this, each level the image is decomposed into the four parts. To do analysis, original signal DWT provides the sufficient information and requires less computation time.

- PCA (Principal Component Analysis) is used to reduce the dimension of the data by means of data compression basics and reveals the most effective low dimensional structure of facial patterns. This reduction in dimensions removes information that is not useful and precisely decomposes the face structure which involves the transformation of a number of possibly correlated variables into a smaller number of orthogonal (uncorrelated) components known as Principal Components. Each face image may be represented as a weighted sum (feature vector) of the Eigen faces, which are stored in a 1D array. It also highlights the differences and similarities amongst the dataset. It is also useful for the extraction of a set of Eigen's faces from the initially inputted images of human faces. And these Eigen faces can also be recombined in the proper ratio to reconstruct the original face image.

- Gabor Wavelet technique is used in image processing where it is separated into two series of one-dimensional ones. This technique is basically used to detect the edges, corners, and blobs of the face image. Gabor functions help to extract the features, especially in texture-based image analysis.

- LDA (Linear Discriminant Analysis) finds most of its applications in appearance based methods. It is considered to be an efficient and qualitative algorithm for selection of features in the applications it is being employed.

But the problem is that individually these techniques are not so efficient and effective. Hence the focus of the work is to implement a hybrid technique for face recognition. After studying previous results the combination of PCA and LDA is chosen as proposed work. The reason behind such decision is whenever LDA is applied; it is applied together with PCA. The dimensions are reduced using PCA and the problem of low power is overcome by LDA as it maximizes power.

\section{PROBLEM FORMULATION}

The face is our primary focus of attention in social intercourses. It plays an important role in providing human identity. Face recognition is a section of pattern recognition in which human visual perception is saved in the computer. This approach is much popular in many of the fields, the main and the important one is recognition or can say as the security or authentication purpose. Many researchers are working on this field for many of the years, many algorithms and techniques are developed to update the traditional systems which are common these days like PCA, LDA, and Gabor etc. But these approaches individually are not that much efficient in some of the cases, so the mixing of the algorithms are done, as an example if PCA approach is used it will provide better results for small datasets. So to overcome these disadvantages the continuous research is processed to get better results. There is one issue too if the algorithms are getting advanced the fake parties are also able to make the algorithm crackable. So there is a need to develop an algorithm which will work as an advanced and modified approach making extraction that much complex that will not be easy to crack up to an extent, so this study gives a proposed study in the field of classification or security.

\section{PROPOSED SYSTEM}

As problems are discussed in the previous section a new approach is proposed for the research work, in which the main concentration will be to extract the features with the PCA and LDA techniques and also with the combined systems. After combine feature extraction of both techniques, finally the classification will be done and the performance will be evaluated.

\section{BLOCK DIAGRAM}

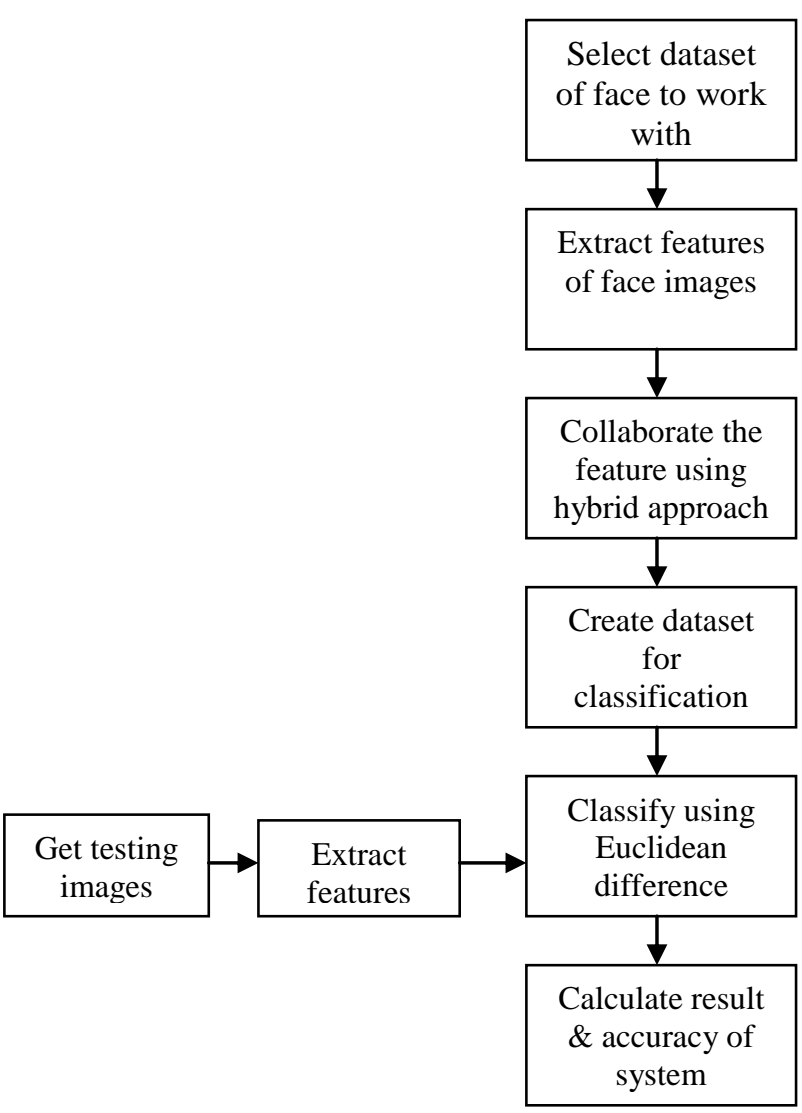

Fig. 1 Block diagram of proposed work 


\section{METHODOLOGY}

1. Select dataset of face to work with for the recognition purpose which will be downloaded from the internet sources generally used for research purposes.

2. Extract features of face images individually and store that in form of matrix.

3. Collaborating the feature using hybrid approach so that further it can be used for the recognition purpose.

4. Create a dataset for classification in which the test images are used which are to classify the accuracy of the system.

5. Extract the same feature of the test dataset so that can be classified by the trained system.

6. Classify using the Euclidean difference between the test dataset and the trained dataset with the hybrid features.

7. Calculate result \& accuracy of the system to evaluate the performance of the system.

\section{RESULTS AND DISCUSSIONS}

Face recognition is a rapidly growing field today for many uses in the fields of biometric authentication, security, and many other areas.

In this, a new method is proposed for the face recognition that is considered to be better than the traditional methods. The proposed method is tested on face database. The figure below represents the original face image for the purpose of face recognition.

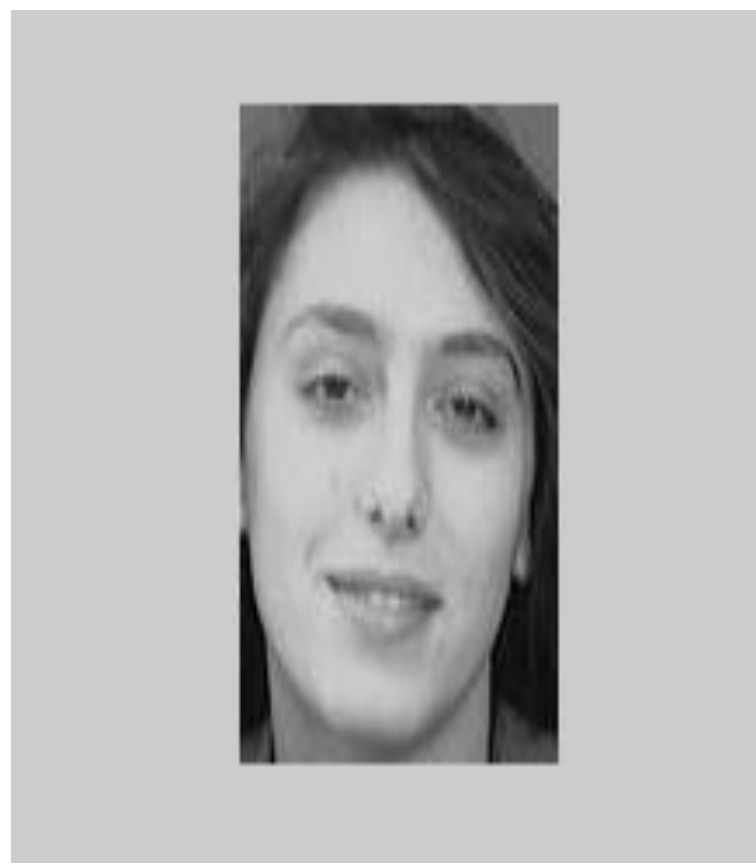

Fig.2 Original face image.

The figure below shows the mean face image after applying PCA on the original image. PCA produces the mean image of the input image.

\section{Mean Face of Dataset}

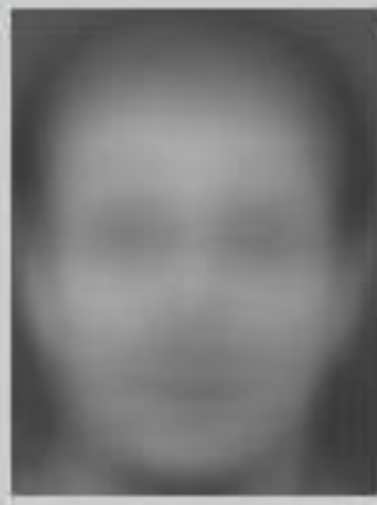

Fig.3 Mean Image obtained after applying PCA

The figure below presents the Eigen face of the original image which is produced by applying LDA on it.

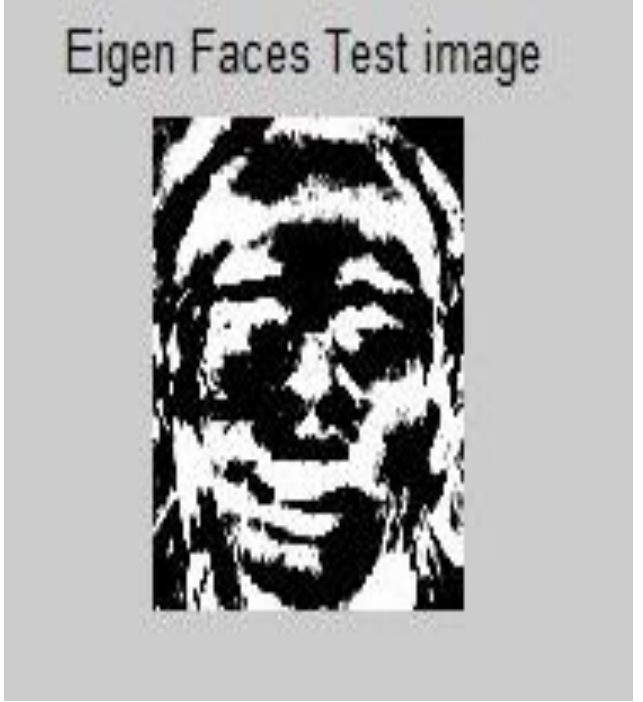

Fig. 4 Eigen face image obtained after applying LDA

Figure 5 asks the user to press the enter button for initializing the testing of datasets.

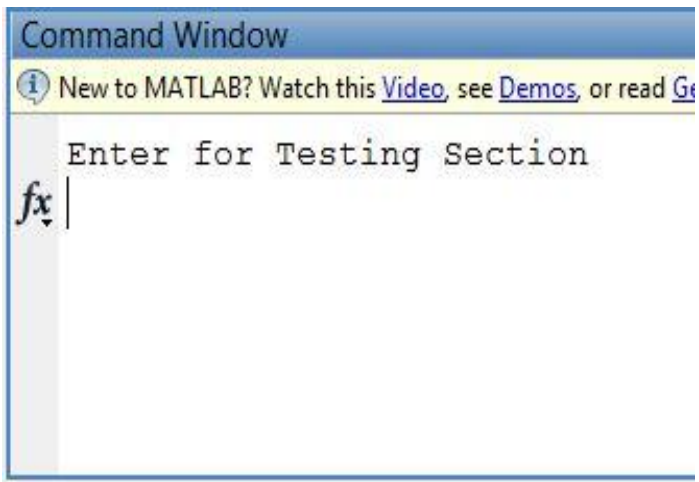

Fig. 5 Initialization of testing datasets 
Figure 6 shows the accuracy of proposed work after applying testing on it. It shows that the accuracy of PCA is 99.3158 and accuracy of LDA is 98.2200 . It shows the accuracy of both of the technique individually.

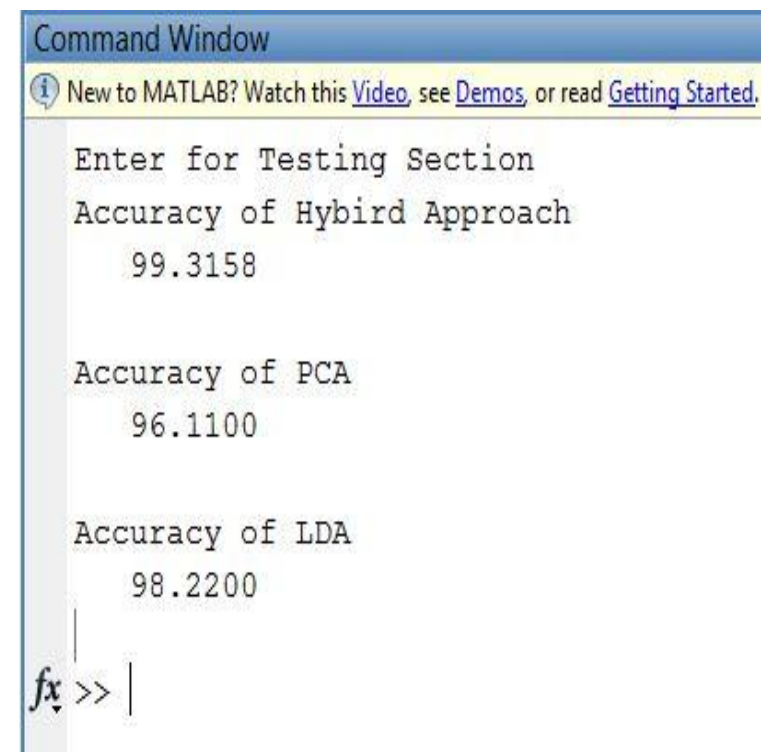

Fig. 6 Individual accuracy of LDA and PCA

The figure below represents the accuracy of proposed work in the graphical form. It shows that after combining LDA and PCA the accuracy achieved is near $99.99 \%$.

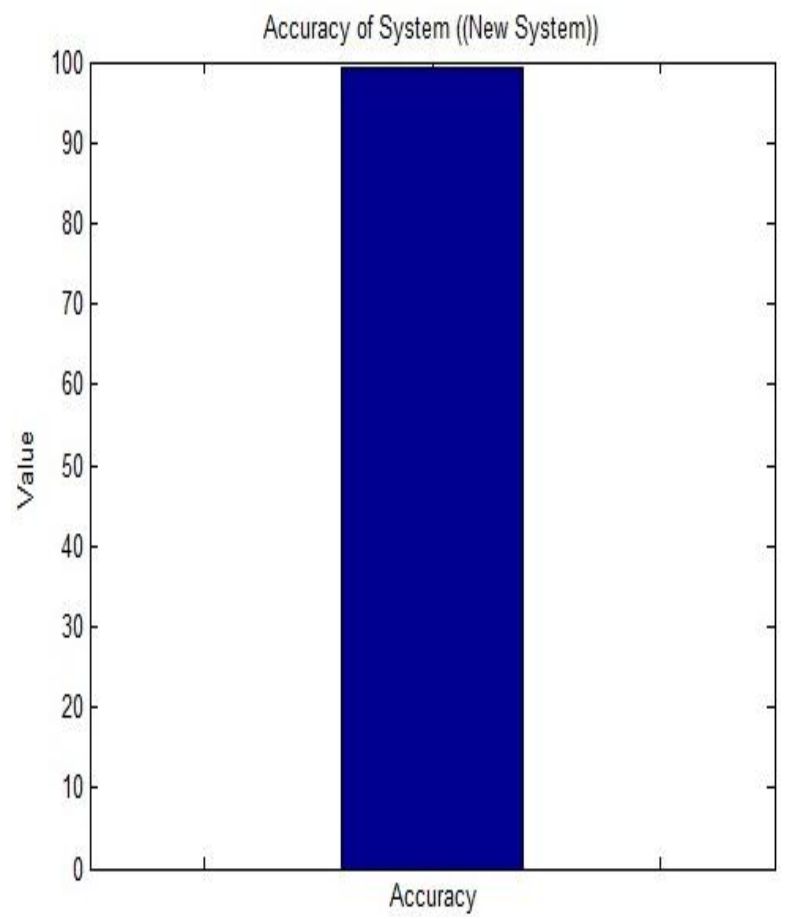

Fig. 7 Accuracy achieved after applying proposed work.

The graph below shows the comparison among LDA, PCA and proposed work. The graph shows that LDA has $95 \%$ approx. accuracy, PCA has $92 \%$ approx. accuracy and accuracy of proposed work is approximately $98.9 \%$.

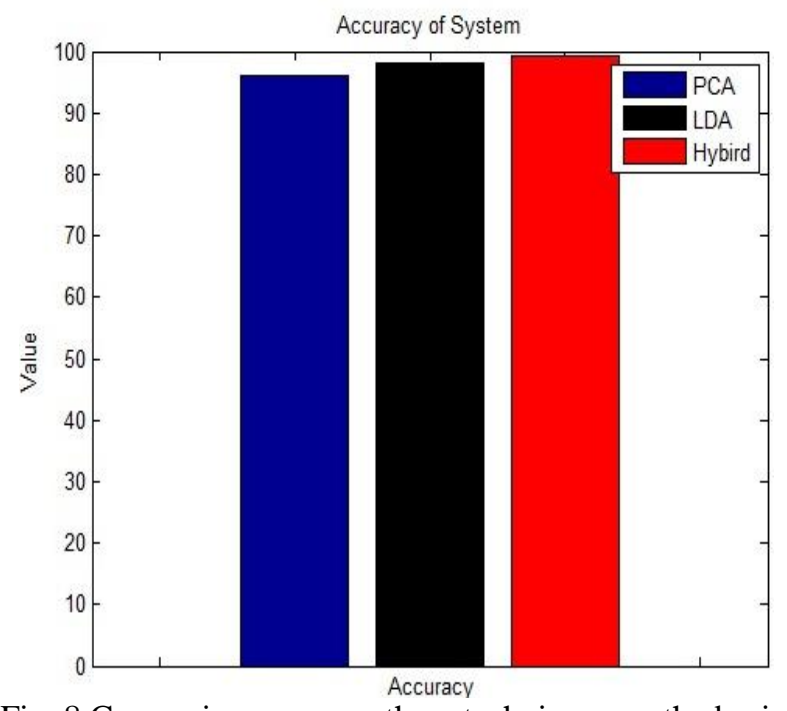

Fig. 8 Comparisons among three techniques on the basis of accuracy

Figure 9 shows the variations between testing samples after applying the proposed hybrid work.

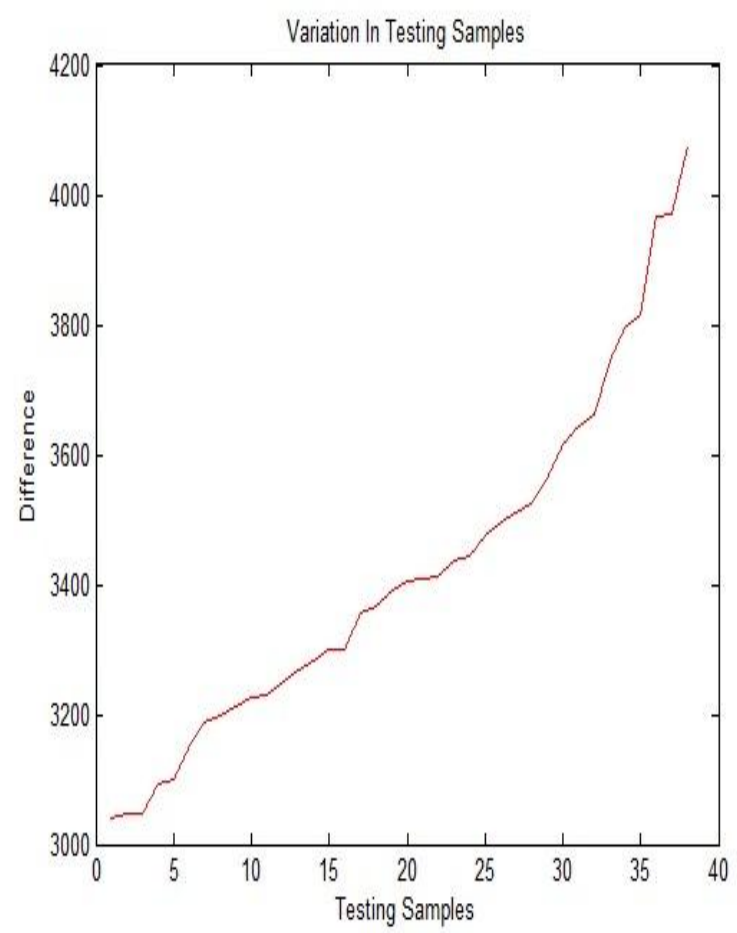

Fig. 9 Variations in testing images after applying hybrid technique

\section{CONCLUSION AND FUTURE SCOPE}

Face recognition systems are widely used biometric systems that play important role in the surveillance systems. This is an efficient technique as the face identification is a unique identification. The features extraction and the classification of the image plays important role in the identification. In this, a new method 
is proposed in which the system is trained after which the testing is done. In this, the feature is extracted using the PCA and LDA and also the combined technique. On the basis of the performance of the system which is measured by varying the number of faces of each subject in the training and test faces. A comparison is performed between proposed and the traditional approach. From the results obtained it is concluded that this proposed method is better than the traditional method for face recognition as this system is more accurate compared to the traditional systems.

In future, this technique can be enhanced further by using some other classifier that can increase the accuracy of the system. Better the extracted features more accurate is the system and thus the efficiency of the system increases. Also, the security of the system shall have increased in order to use this system for authenticate.

\section{REFERENCES}

[1] Firoz Mahmud, "Face recognition using Principle Component Analysis and Linear Discriminant Analysis" IEEE, 21-23 May 2015, Pp 1-4

[2] Abhishree T.M "Face recognition using Gabor Filter based feature extraction with anisotropic diffusion as a pre-processing technique" International conference on Advanced computing technologies, 2015,Pp $312-321$

[3] Mohammad Javed, "Face Recognition using Principle Gabor Filter" International Journal of Advanced Research in Computer Science and Software Engineering, Volume 4, Issue 2, February 2014,Pp 176-180

[4] Ahmad, Faizan,"Image-based Face Detection and Recognition" International Journal of Computer Science, (2012), Volno. 6.

[5] Ben Niua "Two-dimensional Laplacain faces method for face recognition" ELSEVIER, Vol 41 ,2008, Pp 3237 - 3243.

[6] C Nagaraju, "An efficient Facial Features extraction Technique for Face Recognition system Using Local Binary Patterns", International Journal of Innovative Technology and Exploring Engineering (IJITEE), May 2013, Volume-2, Issue-6, May, Pp 74-78.

[7] Grafsgaard,"Automatically Recognizing Facial Expression: Predicting Engagement and Frustration", 2013, Pp. 43-50.

[8] Hehua Chi a, "Facial expression recognition based on cloud model "The International Archives of the Photogrammetric, Remote Sensing and Spatial Information Sciences, Vol. 38, Part II, Pp 124128.

[9] Hiremath,"Face recognition using Eigenface approach." In IDT workshop on interesting results in computer science and engineering, Sweden. 2009.

[10] Jeremiah r. Barr "face recognition from video review", International Journal of Pattern Recognition and Artificial Intelligence,(2012), Volume 26, Issue 05.

[11] Liao, ShengCai, "Face recognition using ordinal features.",In Advances in Biometrics,. Springer, 2006, Pp 40-46.

[12] Mayank Agarwal, "Face Recognition Using Eigen Faces and Artificial Neural Network", International Journal of Computer Theory and Engineering, August, 2010, Vol. 2, No. 4, Pp 624-629.

[13] Mohamed," A new Method for Face Recognition Using Variance Estimation and Feature Extraction."

[14] Nagi Jawad,. "A MATLAB based face recognition system using image processing and neural networks." In 4th International Colloquium on Signal Processing and its Applications, Pp. 83-88.

[15] Navneet Jindal, "Enhanced Face Recognition Algorithm uses PCA with Artificial Neural Networks", International Journal of Advanced Research in Computer Science and Software Engineering, June 2013,Volume 3, Issue 6, Pp 846-872.

[16] N.G.Chitaliya, "An Efficient Method for Face Feature Extraction and Recognition based on Contour let Transform and Principal
Component Analysis using Neural Network", International Journal of Computer Applications ,2010,Volume 6- No.4, Pp 28-32

[17] Pawan Sinha, "Face Recognition by Humans: Nineteen Results All Computer Vision Researchers Should Know About" , Proceedings of the IEEE , (2006), Vol. 94, No. 11, Pp1948-1962.

[18] Ribarić, "Eigen phase-based face recognition: a comparison of phase-information extraction methods." In Nineteenth International Electro technical and Computing Science Conference ERK 2010.

[19] Senior Andrew W, "Face recognition and its application."In BiometricSolutions, Pp. 83-97. Springer US, 2002." Achievements and Challenges in Fingerprint Recognition",Pp 101115

[20] Soninder Singh Garcha ,A robust technique implementation for facial recognition under Eigen feature extraction", Journal of Global Research in Computer Science, 2013 ,Vol 4, No 8.

[21] Sujata G. Bhele, "A Review Paper on Face Recognition Techniques", International Journal of Advanced Research in Computer Engineering \&Technology , October 2012 Volume 1, Issue 8, Pp 338-347

[22] Tayal,Yogesh,"Face Recognition using Eigenface.", International Journal of Emerging Technologies in Computational and Applied Sciences , 2013,Vol 3, no. 1 Pp 50-55.

[23] Teja,"Face recognition using subspaces techniques", Recent Trends In Information Technology (ICRTIT), International Conference, April 2012,Vol 19 issue 21 , Pp103 - 107

[24] Wong, "An efficient algorithm for human face detection and facial feature extraction under different conditions.",Elsevier, 2001, Vol 34. Issue no.10 Pp 1993-2004

[25] Zeynep, "A Study on face, eye detection and gaze estimation." International Journal of Computer Science and Engineering Survey 2, 2011, Vol 3. 